Commun.Fac.Sci.Univ.Ank.Ser. A1 Math. Stat.

Volume 69, Number 1, Pages 941-951(2020)

DOI: $10.31801 /$ cfsuasmas. 628863

ISSN 1303-5991 E-ISSN 2618-6470

http://communications.science.ankara.edu.tr

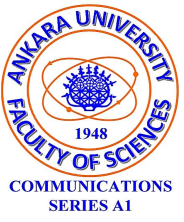

\title{
GENERALIZED DIFFERENCE SEQUENCE SPACES OF FRACTIONAL ORDER DEFINED BY ORLICZ FUNCTIONS
}

\author{
NAZLIM DENIZ ARAL AND MIKAIL ET
}

\begin{abstract}
The main purpose of this paper is to introduce the concepts of $\Delta^{\alpha}$-lacunary statistical convergence of order $\beta(0<\beta \leq 1)$ with the fractional order of $\alpha$ and $\Delta^{\alpha}$-lacunary strongly convergence of order $\beta(0<\beta \leq 1)$ with the fractional order of $\alpha$. We establish some connections between $\Delta^{\alpha}$-lacunary strongly convergence of order $\beta$ and $\Delta^{\alpha}$-lacunary statistical convergence of order $\beta$.
\end{abstract}

\section{INTRODUCTION}

The idea of statistical convergence was given by Zygmund [45] in the first edition of his monograph published in Warsaw in 1935. The concept of statistical convergence was introduced by Steinhaus 42 and Fast 20] and later reintroduced by Schoenberg 38. Over the years and under different names statistical convergence was discussed in the theory of Fourier analysis, Ergodic theory, Number theory, Measure theory, Trigonometric series, Turnpike theory and Banach spaces. Later on it was further investigated from the sequence space point of view and linked with summability theory by Çakallı et al. $([7,[8,9])$. Caserta et al. [10, Çinar et al. [11, Connor [13, Et et al. ([15,,17]), Fridy [22], Fridy and Orhan [23], Mursaleen [33. Salat [41, Mohiuddine et al. ([5], 31]) and many others.

The idea of statistical convergence depends upon the density of subsets of the set $\mathbb{N}$ of natural numbers. The density of a subset $\mathbb{E}$ of $\mathbb{N}$ is defined by

$$
\delta(\mathbb{E})=\lim _{n \rightarrow \infty} \frac{1}{n} \sum_{k=1}^{n} \chi_{\mathbb{E}}(k), \text { provided that the limit exists. }
$$

A sequence $x=\left(x_{k}\right)$ is said to be statistically convergent to $L$ if for every $\varepsilon>0$,

$$
\delta\left(\left\{k \in \mathbb{N}:\left|x_{k}-L\right| \geq \varepsilon\right\}\right)=0 .
$$

Received by the editors: October 03, 2019; Accepted: March 11, 2020.

2010 Mathematics Subject Classification. 40A05, 40C05, 46A45.

Key words and phrases. Difference sequence, statistical convergence, lacunary sequence. 
Recently, Çolak [12] generalized the statistical convergence by ordering the interval $(0,1]$ and defined the statistical convergence of order $\beta$ and strong $p$-Cesàro summability of order $\beta$, where $0<\beta \leq 1$ and $p$ is a positive real number. Şengül and Et $([19], 39])$ generalized the concepts such as lacunary statistical convergence of order $\beta$ and lacunary strong $p$-Cesàro summability of order $\beta$ for sequences of real numbers.

Difference sequence spaces was defined by Kizmaz [27] and the concept was generalized by Et et al. ([14], [18]) as follows:

$$
\Delta^{m}(X)=\left\{x=\left(x_{k}\right):\left(\Delta^{m} x_{k}\right) \in X\right\},
$$

where $X$ is any sequence space, $m \in \mathbb{N}, \Delta^{0} x=\left(x_{k}\right), \Delta x=\left(x_{k}-x_{k+1}\right), \Delta^{m} x=$ $\left(\Delta^{m} x_{k}\right)=\left(\Delta^{m-1} x_{k}-\Delta^{m-1} x_{k+1}\right)$ and so $\Delta^{m} x_{k}=\sum_{v=0}^{m}(-1)^{v}\left(\begin{array}{c}m \\ v\end{array}\right) x_{k+v}$.

If $x \in \Delta^{m}(X)$ then there exists one and only one sequence $y=\left(y_{k}\right) \in X$ such that $y_{k}=\Delta^{m} x_{k}$ and

$$
\begin{gathered}
x_{k}=\sum_{v=1}^{k-m}(-1)^{m}\left(\begin{array}{c}
k-v-1 \\
m-1
\end{array}\right) y_{v}=\sum_{v=1}^{k}(-1)^{m}\left(\begin{array}{c}
k+m-v-1 \\
m-1
\end{array}\right) y_{v-m}, \\
y_{1-m}=y_{2-m}=\cdots=y_{0}=0
\end{gathered}
$$

for sufficiently large $k$, for instance $k>2 m$. After then some properties of difference

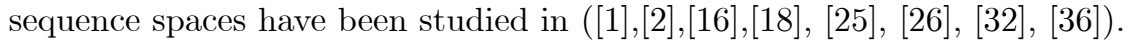

By $\Gamma(r)$, we denote the Gamma function of a real number $r$ and $r \notin\{0,-1,-2,-3, \ldots\}$. By the definition, it can be expressed as an improper integral as:

$$
\Gamma(r)=\int_{0}^{\infty} e^{-t} t^{r-1} d t
$$

From the definition, it is observed that:

(i) For any natural number $n, \Gamma(n+1)=n$ !,

(ii) For any real number $n$ and $n \notin\{0,-1,-2,-3, \ldots\}, \Gamma(n+1)=n \Gamma(n)$,

(iii) For particular cases, we have $\Gamma(1)=\Gamma(2)=1, \Gamma(3)=2$ !,$\Gamma(4)=3$ !, $\ldots$.

For a proper fraction $\alpha$, we define a fractional difference operator $\Delta^{\alpha}: w \rightarrow w$ defined by

$$
\Delta^{\alpha}\left(x_{k}\right)=\sum_{i=0}^{\infty}(-1)^{i} \frac{\Gamma(\alpha+1)}{i ! \Gamma(\alpha-i+1)} x_{k+i}
$$

In particular, we have

$$
\begin{aligned}
\Delta^{\frac{1}{2}} x_{k} & =x_{k}-\frac{1}{2} x_{k+1}-\frac{1}{8} x_{k+2}-\frac{1}{16} x_{k+3}-\frac{5}{128} x_{k+4}-\frac{7}{256} x_{k+5}-\frac{21}{1024} x_{k+6} \cdots \\
\Delta^{-\frac{1}{2}} x_{k} & =x_{k}+\frac{1}{2} x_{k+1}+\frac{3}{8} x_{k+2}+\frac{5}{16} x_{k+3}+\frac{35}{128} x_{k+4}+\frac{63}{256} x_{k+5}+\frac{231}{1024} x_{k+6} \cdots \\
\Delta^{\frac{1}{3}} x_{k} & =x_{k}-\frac{1}{3} x_{k+1}-\frac{1}{9} x_{k+2}-\frac{5}{81} x_{k+3}-\frac{10}{243} x_{k+4}-\frac{22}{729} x_{k+5}-\frac{154}{6561} x_{k+6} \cdots \\
\Delta^{\frac{2}{3}} x_{k} & =x_{k}-\frac{2}{3} x_{k+1}-\frac{1}{9} x_{k+2}-\frac{4}{81} x_{k+3}-\frac{7}{243} x_{k+4}-\frac{14}{729} x_{k+5}-\frac{91}{6561} x_{k+6} \cdots
\end{aligned}
$$


Without loss of generality, we assume throughout that the series defined in (2) is convergent. Moreover, if $\alpha$ is a positive integer, then the infinite sum defined in (2) reduces to a finite sum i.e., $\sum_{i=0}^{\alpha}(-1)^{i} \frac{\Gamma(\alpha+1)}{i ! \Gamma(\alpha-i+1)} x_{k+i}$. In fact, this operator generalized the difference operator introduced by Et and Çolak [14].

Recently, using fractional operator $\Delta^{\alpha}$ (fractional order of $\alpha, \alpha \in \mathbb{R}$ ) Baliarsingh et al. (3, [4, 35]) defined the sequence space $\Delta^{\alpha}(X)$ such as: $\Delta^{\alpha}(X)=$ $\left\{x=\left(x_{k}\right):\left(\Delta^{\alpha} x_{k}\right) \in X\right\}$, where $X$ is any sequence space.

By a lacunary sequence we mean an increasing integer sequence $\theta=\left(k_{r}\right)$ of non-negative integers such that $k_{0}=0$ and $h_{r}=\left(k_{r}-k_{r-1}\right) \rightarrow \infty$ as $r \rightarrow \infty$. The intervals determined by $\theta$ will be denoted by $I_{r}=\left(k_{r-1}, k_{r}\right]$ and the ratio $\frac{k_{r}}{k_{r-1}}$ will be abbreviated by $q_{r}$, and $q_{1}=k_{1}$ for convenience. In recent years, lacunary sequences have been studied in $([7,[8],[9,21], 23], 24], 40])$.

An Orlicz function is a function $M:[0, \infty) \rightarrow[0, \infty)$, which is continuous, nondecreasing and convex with $M(0)=0, M(x)>0$ for $x>0$ and $M(x) \rightarrow \infty$ as $x \rightarrow \infty$.

The study of Orlicz sequence spaces was initiated with a certain specific purpose in Banach space theory. Indeed, Lindberg 29] got interested in Orlicz sequence spaces in connection with finding Banach spaces with symmetric Schauder bases having complementary subspaces isomorphic to $c_{0}$ or $\ell_{p}(0 \leq p<\infty)$. Subsequently, Lindenstrauss and Tzafriri [30] used the idea of Orlicz function to construct the sequence space

$$
\ell_{M}=\left\{x \in w: \sum_{k=1}^{\infty} M\left(\frac{\left|x_{k}\right|}{\rho}\right)<\infty \text { for some } \rho>0\right\} .
$$

The space $\ell_{M}$ with the norm

$$
\|x\|=\inf \left\{\rho>0: \sum_{k=1}^{\infty} M\left(\frac{\left|x_{k}\right|}{\rho}\right) \leq 1\right\}
$$

becomes a Banach space, called an Orlicz sequence space. The space $\ell_{M}$ is closely related to the space $\ell_{p}$ which is an Orlicz sequence space with $M(x)=|x|^{p}$ for $1 \leq p<\infty$. Lindenstrauss and Tzafriri [30] proved that every Orlicz sequence space $\ell_{M}$ contains a subspace isomorphic to $l_{p}(1 \leq p<\infty)$. The Orlicz sequence spaces are the special cases of Orlicz spaces studied in [28].

It is well known that if $M$ is a convex function and $M(0)=0$, then $M(\lambda x) \leq$ $\lambda M(x)$ for all $\lambda$ with $0<\lambda<1$.

Recently, Orlicz sequence spaces were studied by Bhardwaj and Singh [6],Mursaleen et al. (16, 34]), Savaş and Rhoades [37, Tripathy et al. [43] and many others. 


\section{Main Results And PRoOfs}

Definition 1. Let $\theta=\left(k_{r}\right)$ be a lacunary sequence, $\beta \in(0,1]$ and $\alpha$ be a proper fraction. The sequence $x=\left(x_{k}\right)$ is said to be $\Delta^{\alpha}$-lacunary statistically convergent of order $\beta$ of fractional order of $\alpha$ (or $\Delta^{\alpha}\left(S_{\theta}^{\beta}\right)$-convergent to $L$ ) to the number $L$, if there is a real number $L$ such that

$$
\lim _{r \rightarrow \infty} \frac{1}{h_{r}^{\beta}}\left|\left\{k \in I_{r}:\left|\Delta^{\alpha} x_{k}-L\right| \geq \varepsilon\right\}\right|=0
$$

for all $\varepsilon>0$. In this case, we write $x_{k} \rightarrow L\left(\Delta^{\alpha}\left(S_{\theta}^{\beta}\right)\right.$.

The set of all $\Delta^{\alpha}\left(S_{\theta}^{\beta}\right)$-convergent sequences will be denoted by $\Delta^{\alpha}\left(S_{\theta}^{\beta}\right)$. If $\theta=\left(2^{r}\right)$, then we write $\Delta^{\alpha}\left(S^{\beta}\right)$ instead of $\Delta^{\alpha}\left(S_{\theta}^{\beta}\right)$. In the special cases $\theta=\left(2^{r}\right)$ and $\beta=1$, we write $\Delta^{\alpha}(S)$ instead of $\Delta^{\alpha}\left(S_{\theta}^{\beta}\right)$.

In particular, $\Delta^{\alpha}\left(S_{\theta}^{\beta}\right)$-convergence includes many special cases; for example, in case of $\alpha=m \in \mathbb{N}, \beta=1, \Delta^{\alpha}$-lacunary statistical convergence of order $\beta$ reduces to the $\Delta^{m}$-lacunary statistical convergence which was defined and studied by Tripathy and Et [4].

Definition 2. Let $M$ be an Orlicz function, $\theta=\left(k_{r}\right)$ be a lacunary sequence, $\beta \in(0,1], \alpha$ be a proper fraction and $p=\left(p_{k}\right)$ be a sequence of strictly positive real numbers. The sequence $x=\left(x_{k}\right)$ is said to be strongly $\Delta^{\alpha}\left(N_{\theta}^{\beta},(p)\right)$-summable to $L$ with respect to the Orlicz function $M$ (or strongly $\Delta^{\alpha}\left(N_{\theta}^{\beta}, M,(p)\right)$-summable to $L)$, if there is a real number $L$ such that

$$
\lim _{r \rightarrow \infty} \frac{1}{h_{r}^{\beta}} \sum_{k \in I_{r}}\left[M\left(\frac{\left|\Delta^{\alpha} x_{k}-L\right|}{\rho}\right)\right]^{p_{k}}=0,
$$

for all $\varepsilon>0$ and some $\rho>0$. In this case, we write $x_{k} \rightarrow L\left(\Delta^{\alpha}\left(N_{\theta}^{\beta}, M,(p)\right)\right)$.

The set of all $\Delta^{\alpha}\left(N_{\theta}^{\beta}, M,(p)\right)$-summable sequences will be denoted by $\Delta^{\alpha}\left(N_{\theta}^{\beta}, M,(p)\right)$. In the special cases $M(x)=x, p_{k}=p$ for each $k \in \mathbb{N}$, we obtain the set $\Delta^{\alpha}\left(N_{\theta}^{\beta}, p\right)$. If $\theta=\left(2^{r}\right), M(x)=x, p_{k}=1$ for each $k \in \mathbb{N}$ and $\beta=1$, then we write $\Delta^{\alpha}\left(\left|\sigma_{1}\right|\right)$ instead of $\Delta^{\alpha}\left(N_{\theta}^{\beta}, p\right)$ and say that $x=\left(x_{k}\right)$ is strongly $\Delta^{\alpha}$-Cesàro summable to $L$.

The proof the following theorems are straightforward, so we choose to state these results without proof.

Theorem 3. Let $\theta=\left(k_{r}\right)$ be a lacunary sequence, $\beta \in(0,1]$, $\alpha$ be a proper fraction and $x=\left(x_{k}\right), y=\left(y_{k}\right)$ are sequences of real numbers, then

i) If $x_{k} \rightarrow L\left(\Delta^{\alpha}\left(S_{\theta}^{\beta}\right)\right)$ and $c \in \mathbb{C}$, then $c x_{k} \rightarrow c L\left(\Delta^{\alpha}\left(S_{\theta}^{\beta}\right)\right)$.

ii) If $x_{k} \rightarrow L_{1}\left(\Delta^{\alpha}\left(S_{\theta}^{\beta}\right)\right)$ and $y_{k} \rightarrow L_{2}\left(\Delta^{\alpha}\left(S_{\theta}^{\beta}\right)\right)$, then $\left(x_{k}+y_{k}\right) \rightarrow\left(L_{1}+L_{2}\right)\left(\Delta^{\alpha}\left(S_{\theta}^{\beta}\right)\right)$. 
Theorem 4. Let the sequence $\left(p_{k}\right)$ be bounded, then the sequence space $\Delta^{\alpha}\left(N_{\theta}^{\beta}, M,(p)\right)$ is a linear space over the set of complex numbers.

Theorem 5. If a $\Delta^{\alpha}$-bounded sequence (that is $x \in \Delta^{\alpha}\left(\ell_{\infty}\right)$ ) is $\Delta^{\alpha}$-statistically convergent to $L$ then it is strongly $\Delta^{\alpha}$-Cesàro summable to $L$.

Proof. Suppose that $x \in \Delta^{\alpha}\left(\ell_{\infty}\right) \cap \Delta^{\alpha}(S)$ with $x_{k} \rightarrow L\left(\Delta^{\alpha}(S)\right.$. Without loss of generality we may assume that $L=0$. Set $K=\left\|\Delta^{\alpha} x\right\|_{\infty}$. Let $\varepsilon>0$ be given and choose $N_{\varepsilon}$ such that $\frac{1}{n}\left|\left\{k \leq n:\left|\Delta^{\alpha} x_{k}\right| \geq \frac{\varepsilon}{2}\right\}\right|<\frac{\varepsilon}{2 K}$ for all $n>N_{\varepsilon}$. Now, we get

$$
\frac{1}{n} \sum_{k=1}^{n}\left|\Delta^{\alpha} x_{k}\right|=\frac{1}{n} \sum_{\substack{1 \leq k \leq n \\\left|\Delta^{\alpha} x_{k}\right| \geq \frac{\varepsilon}{2}}}\left|\Delta^{\alpha} x_{k}\right|+\frac{1}{n} \sum_{\substack{1 \leq k \leq n \\\left|\Delta^{\alpha} x_{k}\right|<\frac{\varepsilon}{2}}}\left|\Delta^{\alpha} x_{k}\right| \leq \frac{1}{n} \frac{n \varepsilon}{2 K} K+\frac{n}{n} \frac{\varepsilon}{2}=\varepsilon .
$$

for all $n>N_{\varepsilon}$. Thus $\lim \frac{1}{n} \sum_{k=1}^{n}\left|\Delta^{\alpha} x_{k}\right|=0$ which means that $x \in \Delta^{\alpha}\left(\left|\sigma_{1}\right|\right)$.

Converse of Theorem 5 does not holds. For this choose $\alpha=1$, then the sequence $x=(0,-1,-1,-2,-2,-3-, 3,-4,-4, \ldots)$ belongs to $\Delta\left(\left|\sigma_{1}\right|\right)$ and does not belong to $\Delta(S)$.

Theorem 6. $\Delta^{\alpha}\left(N_{\theta}^{\beta}, p\right)$ is a Banach space normed by

$$
\|x\|_{\Delta_{1}^{\alpha}}=\sum_{i=1}^{\infty}\left|x_{i}\right|+\sup _{r}\left(\frac{1}{h_{r}^{\beta}} \sum_{k \in I_{r}}\left|\Delta^{\alpha} x_{k}\right|^{p}\right)^{1 / p}, 1 \leq p<\infty
$$

and a complete $p$-normed space for $0<p<1$ by

$$
\|x\|_{\Delta_{2}^{\alpha}}=\sum_{i=1}^{\infty}\left|x_{i}\right|+\sup _{r} \frac{1}{h_{r}^{\beta}} \sum_{k \in I_{r}}\left|\Delta^{\alpha} x_{k}\right|^{p}=0
$$

Proof. Proof follows from Theorem 3 [4] and Theorem 2.4 [39].

Theorem 7. $\Delta^{\alpha}\left(N_{\theta}^{\beta}, p\right)$ is a $B K-$ space normed by $(3)$.

Proof. Omitted.

Theorem 8. Let $\theta=\left(k_{r}\right)$ be a lacunary sequence, $\beta \in(0,1], \alpha$ be a proper fraction and $p$ be a fixed positive real number, then

i) If $x_{k} \rightarrow L\left(\Delta^{\alpha}\left(N_{\theta}^{\beta}, p\right)\right)$, then $x_{k} \rightarrow L\left(\Delta^{\alpha}\left(S_{\theta}^{\beta}\right)\right)$ and the inclusion is strict,

ii) (24]) If $x \in \Delta^{\alpha}\left(\ell_{\infty}\right)$ and $x_{k} \rightarrow L\left(\Delta^{\alpha}\left(S_{\theta}\right)\right)$, then $x_{k} \rightarrow L\left(\Delta^{\alpha}\left(N_{\theta}, p\right)\right)$. 
Proof. The inclusion part of the proof is easy. In order to establish "the inclusion is strict", let $\theta$ be given, choose $\alpha=m, \beta=1, p=1$ and define a sequence $x=\left(x_{k}\right)$ by $\Delta^{m} x$ to be $1,2, \ldots,\left[\sqrt{h_{r}}\right]$ at the first $\left[\sqrt{h_{r}}\right]$ integers in $I_{r}$, and $\Delta^{m} x_{k}=0$ otherwise (5)

It is clear that $x$ is not $\Delta^{m}$-bounded. Since

$$
\frac{1}{h_{r}}\left|\left\{k \in I_{r}:\left|\Delta^{m} x_{k}-0\right| \geq \varepsilon\right\}\right|=\frac{\left[\sqrt{h_{r}}\right]}{h_{r}} \rightarrow 0, \text { as } r \rightarrow \infty
$$

and

$$
\frac{1}{h_{r}} \sum_{k \in I_{r}}\left|\Delta^{m} x_{k}-0\right|=\frac{\left[\sqrt{h_{r}}\right]\left(\left[\sqrt{h_{r}}\right]+1\right)}{2 h_{r}} \rightarrow \frac{1}{2}, \text { as } r \rightarrow \infty .
$$

From (1) we have $x \in \Delta^{m}\left(S_{\theta}\right), x_{k} \notin \Delta^{m}\left(N_{\theta}\right)$.

Theorem 9. Let $0<\alpha \leq 1$ and $\theta=\left(k_{r}\right)$ be a lacunary sequence. If $\liminf _{r} q_{r}>1$, then $\Delta^{\alpha}\left(S^{\beta}\right) \subset \Delta^{\alpha}\left(S_{\theta}^{\beta}\right)$.

Proof. Suppose that $\liminf _{r} q_{r}>1$; then there exists a $\delta>0$ such that $q_{r} \geq 1+\delta$ for sufficiently large $r$, which implies that

$$
\frac{h_{r}}{k_{r}} \geq \frac{\delta}{1+\delta} \Longrightarrow\left(\frac{h_{r}}{k_{r}}\right)^{\beta} \geq\left(\frac{\delta}{1+\delta}\right)^{\beta} \Longrightarrow \frac{1}{k_{r}^{\beta}} \geq \frac{\delta^{\beta}}{(1+\delta)^{\beta}} \frac{1}{h_{r}^{\beta}} .
$$

If $x_{k} \rightarrow L\left(\Delta^{\alpha}\left(S^{\beta}\right)\right)$, then for every $\varepsilon>0$ and for sufficiently large $r$, we have

$$
\begin{aligned}
\frac{1}{k_{r}^{\beta}}\left|\left\{k \leq k_{r}:\left|\Delta^{\alpha} x_{k}-L\right| \geq \varepsilon\right\}\right| & \geq \frac{1}{k_{r}^{\beta}}\left|\left\{k \in I_{r}:\left|\Delta^{\alpha} x_{k}-L\right| \geq \varepsilon\right\}\right| \\
& \geq \frac{\delta^{\beta}}{(1+\delta)^{\beta}} \frac{1}{h_{r}^{\beta}}\left|\left\{k \in I_{r}:\left|\Delta^{\alpha} x_{k}-L\right| \geq \varepsilon\right\}\right| ;
\end{aligned}
$$

So $x \in \Delta^{\alpha}\left(S_{\theta}^{\beta}\right)$.

Theorem 10. Let $0<\alpha \leq 1$ and $\theta=\left(k_{r}\right)$ be a lacunary sequence. If $\lim \sup _{r} q_{r}<$ $\infty$, then $\Delta^{\alpha}\left(S_{\theta}^{\beta}\right) \subset \Delta^{\alpha}\left(S^{\beta}\right)$.

Proof. Omitted.

In the following theorems, assume that the sequence $p=\left(p_{k}\right)$ is bounded and $0<h=\inf _{k} p_{k} \leq p_{k} \leq \sup _{k} p_{k}=H<\infty$.

Theorem 11. Let $\beta, \gamma \in(0,1]$ be real numbers such that $\beta \leq \gamma, M$ be an Orlicz function and $\theta=\left(k_{r}\right)$ be a lacunary sequence, then $\Delta^{\alpha}\left(N_{\theta}^{\beta}(M,(p)) \subset \Delta^{\alpha}\left(S_{\theta}^{\gamma}\right)\right.$. 
Proof. Let $x \in \Delta^{\alpha}\left(N_{\theta}^{\beta}(M,(p)), \varepsilon>0\right.$ be given and $\sum_{1}$ and $\sum_{2}$ denote the sums over $k \in I_{r},\left|\Delta^{\alpha} x_{k}-L\right| \geq \varepsilon$ and $\left|\Delta^{\alpha} x_{k}-L\right|<\varepsilon$ respectively. As $h_{r}^{\beta} \leq h_{r}^{\gamma}$ for each $r$, we have

$$
\begin{aligned}
& \frac{1}{h_{r}^{\beta}} \sum_{k \in I_{r}}\left[M\left(\frac{\left|\Delta^{\alpha} x_{k}-L\right|}{\rho}\right)\right]^{p_{k}} \geq \frac{1}{h_{r}^{\gamma}}\left[\begin{array}{c}
\sum_{1}\left[M\left(\frac{\left|\Delta^{\alpha} x_{k}-L\right|}{\rho}\right)\right]^{p_{k}} \\
+\sum_{2}\left[M\left(\frac{\left|\Delta^{\alpha} x_{k}-L\right|}{\rho}\right)\right]^{p_{k}}
\end{array}\right] \\
& \geq \frac{1}{h_{r}^{\gamma}}\left[\sum_{1} M\left(\frac{\varepsilon}{\rho}\right)\right]^{p_{k}} \\
& \geq \frac{1}{h_{r}^{\gamma}} \sum_{1} \min \left(\left[M\left(\varepsilon_{1}\right)\right]^{h},\left[M\left(\varepsilon_{1}\right)\right]^{H}\right), \quad \varepsilon_{1}=\frac{\varepsilon}{\rho} \\
& \geq \frac{1}{h_{r}^{\gamma}}\left|\left\{k \in I_{r}:\left|\Delta^{\alpha} x_{k}-L\right| \geq \varepsilon\right\}\right| \\
& \times \min \left(\left[M\left(\varepsilon_{1}\right)\right]^{h},\left[M\left(\varepsilon_{1}\right)\right]^{H}\right) .
\end{aligned}
$$

As $x \in \Delta^{\alpha}\left(N_{\theta}^{\beta}(M,(p))\right.$, the left hand side of the above inequality tends to zero as $r \rightarrow \infty$. Therefore, the right hand side of the above inequality tends to zero as $r \rightarrow \infty$, hence $x \in \Delta^{\alpha}\left(S_{\theta}^{\gamma}\right)$.

Corollary 12. Let $0<\beta \leq 1, M$ be an Orlicz function and $\theta=\left(k_{r}\right)$ be a lacunary sequence, then $\Delta^{\alpha}\left(N_{\theta}^{\beta}(M,(p)) \subset \Delta^{\alpha}\left(S_{\theta}^{\beta}\right)\right.$.

Theorem 13. Let $M$ be an Orlicz function, $x=\left(x_{k}\right)$ be a $\Delta^{\alpha}$-bounded sequence and $\theta=\left(k_{r}\right)$ be a lacunary sequence. If $\lim _{r \rightarrow \infty} \frac{h_{r}}{h_{r}^{\beta}}=1$, then $x \in \Delta^{\alpha}\left(S_{\theta}^{\beta}\right) \Rightarrow x \in$ $\Delta^{\alpha}\left(N_{\theta}^{\beta}(M,(p))\right.$.

Proof. Suppose that $x=\left(x_{k}\right)$ be a $\Delta^{\alpha}$-bounded sequence, that is $x \in \Delta^{\alpha}\left(\ell_{\infty}\right)$ and $x_{k} \rightarrow L\left(\Delta^{\alpha}\left(S_{\theta}^{\beta}\right)\right)$. As $x \in \Delta^{\alpha}\left(\ell_{\infty}\right)$, then there is a constant $T>0$ such that $\left|\Delta^{\alpha} x_{k}\right| \leq T$. Given $\varepsilon>0$, we have

$$
\begin{aligned}
\frac{1}{h_{r}^{\beta}} \sum_{k \in I_{r}}\left[M\left(\frac{\left|\Delta^{\alpha} x_{k}-L\right|}{\rho}\right)\right]^{p_{k}}= & \frac{1}{h_{r}^{\beta}} \sum_{1}\left[M\left(\frac{\left|\Delta^{\alpha} x_{k}-L\right|}{\rho}\right)\right]^{p_{k}} \\
& +\frac{1}{h_{r}^{\beta}} \sum_{2}\left[M\left(\frac{\left|\Delta^{\alpha} x_{k}-L\right|}{\rho}\right)\right]^{p_{k}} \\
\leq & \frac{1}{h_{r}^{\beta}} \sum_{1} \max \left\{\left[\left[M\left(\frac{T}{\rho}\right)\right]^{h},\left[M\left(\frac{T}{\rho}\right)\right]^{H}\right]\right\}
\end{aligned}
$$




$$
\begin{aligned}
& +\frac{1}{h_{r}^{\beta}} \sum_{2} \max \left[M\left(\frac{\varepsilon}{\rho}\right)\right]^{p_{k}} \\
\leq & \max \left\{[M(K)]^{h},[M(K)]^{H}\right\} \\
& \times \frac{1}{h_{r}^{\beta}}\left|\left\{k \in I_{r}:\left|\Delta^{\alpha} x_{k}-L\right| \geq \varepsilon\right\}\right| \\
& +\frac{h_{r}}{h_{r}^{\beta}} \max \left\{\left[M\left(\varepsilon_{1}\right)\right]^{h},\left[M\left(\varepsilon_{1}\right)\right]^{H}\right\}, \\
\frac{T}{\rho}= & K, \quad \frac{\varepsilon}{\rho}=\varepsilon_{1} .
\end{aligned}
$$

Hence $x \in \Delta^{\alpha}\left(N_{\theta}^{\beta}(M,(p))\right.$.

Theorem 14. If $\lim p_{k}>0$ and $x=\left(x_{k}\right)$ is strongly $\Delta^{\alpha}\left(N_{\theta}^{\beta}(M,(p))\right.$-summable to $L$ with respect to the Orlicz function $M$, then that limit $L$ is unique.

Proof. Let $\lim p_{k}=s>0$. Suppose that $x_{k} \rightarrow L\left(\Delta^{\alpha}\left(N_{\theta}^{\beta}, p\right)\right)$ and $x_{k} \rightarrow L_{1}\left(\Delta^{\alpha}\left(N_{\theta}^{\beta}, p\right)\right)$. Then we have

$$
\lim _{r \rightarrow \infty} \frac{1}{h_{r}^{\beta}} \sum_{k \in I_{r}}\left[M\left(\frac{\left|\Delta^{\alpha} x_{k}-L\right|}{\rho_{1}}\right)\right]^{p_{k}}=0, \text { for some } \rho_{1}>0
$$

and

$$
\lim _{r \rightarrow \infty} \frac{1}{h_{r}^{\beta}} \sum_{k \in I_{r}}\left[M\left(\frac{\left|\Delta^{\alpha} x_{k}-L_{1}\right|}{\rho_{2}}\right)\right]^{p_{k}}=0, \text { for some } \rho_{2}>0 .
$$

We define the $\rho=\max \left(2 \rho_{1}, 2 \rho_{2}\right)$. As $M$ is nondecreasing and convex, we have

$$
\begin{aligned}
\frac{1}{h_{r}^{\beta}} \sum_{k \in I_{r}}\left[M\left(\frac{L-L_{1}}{\rho}\right)\right]^{p_{k}} \leq & \frac{D}{h_{r}^{\beta}} \sum_{k \in I_{r}} \frac{1}{2^{p_{k}}} \\
& \times\left(\left[M\left(\frac{\left|\Delta^{\alpha} x_{k}-L\right|}{\rho_{1}}\right)\right]^{p_{k}}+\left[M\left(\frac{\left|\Delta^{\alpha} x_{k}-L_{1}\right|}{\rho_{2}}\right)\right]^{p_{k}}\right) \\
\leq & \frac{D}{h_{r}^{\beta}} \sum_{k \in I_{r}}\left[M\left(\frac{\left|\Delta^{\alpha} x_{k}-L\right|}{\rho_{1}}\right)\right]^{p_{k}} \\
& +\frac{D}{h_{r}^{\beta}} \sum_{k \in I_{r}}\left[M\left(\frac{\left|\Delta^{\alpha} x_{k}-L_{1}\right|}{\rho_{2}}\right)\right]^{p_{k}} \\
\rightarrow & 0, \quad(r \rightarrow \infty),
\end{aligned}
$$

where $\sup _{k} p_{k}=H$ and $D=\max \left(1,2^{H-1}\right)$. Hence,

$$
\lim _{r \rightarrow \infty} \frac{1}{h_{r}^{\beta}} \sum_{k \in I_{r}}\left[M\left(\frac{L-L_{1}}{\rho}\right)\right]^{p_{k}}=0 .
$$


As $\lim _{k \rightarrow \infty} p_{k}=s$, we have

$$
\lim _{k \rightarrow \infty}\left[M\left(\frac{\left|L-L_{1}\right|}{\rho}\right)\right]^{p_{k}}=\left[M\left(\frac{\left|L-L_{1}\right|}{\rho}\right)\right]^{s}
$$

and so $L=L_{1}$. Thus, the limit is unique.

\section{REFERENCES}

[1] Altınok, H., Et, M., Çolak, R., Some remarks on generalized sequence space of bounded variation of sequences of fuzzy numbers, Iran. J. Fuzzy Syst., 11(5) (2014), 39-46, 109.

[2] Aral, N. D., Et, M., On lacunary statistical convergence of order $\beta$ of difference sequences of fractional order, International Conference of Mathematical Sciences, (ICMS 2019), Maltepe University, Istanbul, Turkey.

[3] Baliarsingh, P., Some new difference sequence spaces of fractional order and their dual spaces, Appl. Math. Comput., 219(18) (2013), 9737-9742.

[4] Baliarsingh, P., Kadak, U., Mursaleen, M., On statistical convergence of difference sequences of fractional order and related Korovkin type approximation theorems, Quaest. Math., 41(8) (2018), 1117-1133.

[5] Belen, C., Mohiuddine, S. A., Generalized weighted statistical convergence and application, Appl. Math. Comput., 219 (2013), 9821-9826.

[6] Bhardwaj, V. K., Singh, N., Some sequences defined by Orlicz functions, Demonstratio Math., 33(3) (2000), 571-582.

[7] Çakallı, H., Lacunary statistical convergence in topological groups, Indian J. Pure Appl. Math., 26(2) (1995), 113-119.

[8] Çakallı, H., Aras, C. G., Sönmez, A., Lacunary statistical ward continuity, AIP Conf. Proc., 1676, 020042 (2015); http://dx.doi.org/10.1063/1.4930468.

[9] Çakall, H., Kaplan, H., A variation on lacunary statistical quasi Cauchy sequences, Commun. Fac. Sci. Univ. Ank. Sér. A1 Math. Stat., 66(2) (2017), 71-79.

[10] Caserta, A., Di Maio, G., Kočinac, L. D. R., Statistical convergence in function spaces, Abstr. Appl. Anal., 2011, Art. ID 420419, 11 pp.

[11] Çınar, M., Karakaş, M., Et, M., On pointwise and uniform statistical convergence of order $\alpha$ for sequences of functions, Fixed Point Theory And Applications, Article Number: 33, 2013.

[12] Çolak, R., Statistical convergence of order $\alpha$, Modern Methods in Analysis and Its Applications, New Delhi, India: Anamaya Pub., (2010) 121-129.

[13] Connor, J. S., The statistical and strong p-Cesaro convergence of sequences, Analysis, 8 (1988), 47-63.

[14] Et, M., Çolak, R., On some generalized difference sequence spaces, Soochow J. Math., 21(4) (1995), 377-386 .

[15] Et, M., Tripathy, B. C., Dutta, A. J., On pointwise statistical convergence of order $\alpha$ of sequences of fuzzy mappings, Kuwait J. Sci., 41(3) (2014), 17-30.

[16] Et, M., Mursaleen, M., Işı, M., On a class of fuzzy sets defined by Orlicz functions, Filomat, $27(5)$ (2013), 789-796.

[17] Et, M., Çolak, R., Altin, Y., Strongly almost summable sequences of order $\alpha$, Kuwait J. Sci., $41(2)(2014), 35-47$.

[18] Et, M. , Nuray, F., $\Delta^{m}$-Statistical convergence, Indian J. Pure appl. Math. 32(6) (2001), $961-969$.

[19] Et, M., Şengül, H., Some Cesaro-Type Summability Spaces of Order alpha and Lacunary Statistical Convergence of Order $\alpha$, Filomat, 28(8) (2014), 1593-1602.

[20] Fast, H., Sur la convergence statistique, Colloq. Math., 2 (1951), 241-244. 
[21] Freedman, A. R., Sember, J. J., Raphael, M., Some Cesaro-type summability spaces, Proc. Lond. Math. Soc., 37(3) (1978), 508-520.

[22] Fridy, J., On statistical convergence, Analysis, 5 (1985), 301-313.

[23] Fridy, J., Orhan, C., Lacunary statistical convergence, Pacific J. Math., 160 (1993), 43-51.

[24] Kadak, U., Generalized lacunary statistical difference sequence spaces of fractional order, Int. J. Math. Math. Sci., 2015, Art. ID 984283, 6 pp.

[25] Kadak, U., Mohiuddine, S. A., Generalized statistically almost convergence based on the difference operator which includes the (p, q)-gamma function and related approximation theorems, Results Math., 73(1) (2018), Article 9.

[26] Karakaş, M., Et, M., Karakaya, V., Some geometric properties of a new difference sequence space involving lacunary sequences, Acta Math. Sci. Ser. B (Engl. Ed.), 33(6) (2013), 17111720 .

[27] Kizmaz, H., On certain sequence spaces, Canad. Math. Bull., 24(2) (1981), 169-176.

[28] Krasnosel'skii, M. A.,Rutickii, Y. B., Convex Functions and Orlicz Spaces, Groningen, Netherlands, (1961).

[29] Lindberg, K., On subspaces of Orlicz sequence spaces, Studia Math., 45 (1973), 119-146.

[30] Lindenstrauss, J., Tzafriri, T., On Orlicz sequence spaces, Israel J. Math., 10 (1971), 379-390.

[31] Mohiuddine, S. A., Alamri, B. A. S., Generalization of equi-statistical convergence via weighted lacunary sequence with associated Korovkin and Voronovskaya type approximation theorems, Rev. R. Acad. Cienc. Exactas Fis. Nat. Ser. A Math. RACSAM, 113(3) (2019), 1955-1973.

[32] Mohiuddine, S. A., Asiri, A., Hazarika, B., Weighted statistical convergence through difference operator of sequences of fuzzy numbers with application to fuzzy approximation theorems, Int. J. Gen. Syst., 48(5) (2019), 492-506.

[33] Mursaleen, M., $\lambda$ - statistical convergence, Math. Slovaca, 50(1) (2012), 111 -115.

[34] Mursaleen, M., Khan, Q. A., Chishti, T. A., Some new convergent sequences spaces defined by Orlicz functions and statistical convergence, Ital. J. Pure Appl. Math., 9 (2001), 25-32.

[35] Nayak, L., Et, M., Baliarsingh, P., On certain generalized weighted mean fractional difference sequence spaces, Proc. Nat. Acad. Sci. India Sect. A, 89(1) (2019), 163-170.

[36] Savaş, E., Et, M., On $\left(\Delta_{\lambda}^{m}, I\right)$-statistical convergence of order $\alpha$, Period. Math. Hungar., 71(2) (2015), 135-145.

[37] Savaş, E., Rhoades, B. E., On some new sequence spaces of invariant means defined by Orlicz functions, Math. Inequal. Appl., 5(2) (2002), 271-281.

[38] Schoenberg, I. J., The integrability of certain functions and related summability methods, Amer. Math. Monthly, 66 (1959), 361-375.

[39] Şengül, H., Et, M., On lacunary statistical convergence of order $\alpha$, Acta Mathematica Scientia, 34(2) 473-482.

[40] Srivastava, H. M., Et, M., Lacunary statistical convergence and strongly lacunary summable functions of order $\alpha$, Filomat, 31(6) (2017), 1573-1582.

[41] Salat, T., On statistically convergent sequences of real numbers, Math. Slovaca, 30 (1980), 139-150.

[42] Steinhaus, H., Sur la convergence ordinaire et la convergence asymptotique, Colloq. Math., 2 (1951), 73-74

[43] Tripathy, B. C., Hazarika, B., Some $I$-convergent sequence spaces defined by Orlicz functions, Acta Math. Appl. Sin. Engl. ser., 27(1) (2011), 149-154.

[44] Tripathy, B. C., Et, M., On generalized difference lacunary statistical convergence, Studia Univ. Babeş-Bolyai Math., 50(1) (2005), 119-130.

[45] Zygmund, A., Trigonometric Series, Cambridge University Press, Cambridge, UK, 1979. 
Current address: Nazlım Deniz ARAL: Department of Mathematics, Faculty of Arts and Sciences, Bitlis Eren University, Bitlis, 13000, Turkey.

E-mail address: ndaral@beu.edu.tr

ORCID Address: https://orcid.org/0000-0002-8984-2620

Current address: Mikail ET: Department of Mathematics, Faculty of Science, Frrat University, Elazı̆̆, 23119, Turkey.

E-mail address: mikailet68@gmail.com

ORCID Address: https://orcid.org/0000-0001-8292-7819 